\title{
1 Decoding differential gene expression
}

\author{
2 Shinya Tasaki ${ }^{1 *}$, Chris Gaiteri $^{1}$, Sara Mostafavi ${ }^{2}$, Yanling Wang ${ }^{1}$ \\ $3 \quad{ }^{1}$ Rush Alzheimer's Disease Center, Rush University Medical Center, Chicago IL, USA \\ $4 \quad{ }^{2}$ University of British Columbia, Vancouver, British Columbia, Canada \\ $5 *$ corresponding author
}

\section{Abstract}

7 Identifying the molecular mechanisms that control differential gene expression (DE) is a major goal of basic and disease 8 biology. Combining the strengths of systems biology and deep learning in a model called DEcode, we are able to predict 9 DE more accurately than traditional sequence-based methods, which do not utilize systems biology data. To determine 10 the biological origins of this accuracy, we identify the most predictive regulators and types of regulatory interactions in 11 DEcode, contrasting their roles across many human tissues. Diverse systems biology, ontological and disease-related 12 assessments all point to the predominant influence of post-translational RNA-binding factors on DE. Through the 13 combinatorial gene regulation that is captured in DEcode, it is even possible to predict relatively subtle person-to-person 14 variation in gene expression. We demonstrate the broad applicability of these clinically-relevant predictions by predicting 15 drivers of aging throughout the human lifespan, gene coexpression relationships on a genome-wide scale, and frequent 16 DE in diverse conditions. Researchers can freely access DEcode to utilize genomic big data in identifying influential 17 molecular mechanisms for any human expression data - www.differentialexpression.org.

\section{Introduction}

19 While all human cells share DNA sequences, gene regulation differs among cell types and developmental stages, and in 20 response to environmental cues and stimuli. Accordingly, when gene expression is not properly regulated, cellular 21 homeostasis can be perturbed, often affecting cell function and leading to disease ${ }^{1}$. These distinctions between cell 22 states are observed as differential expression (DE) of gene transcripts. DE have been cataloged for tens of thousands of 23 gene expression datasets, in the context of distinctions between species, organs, and conditions. Despite the important 
24 and pervasive nature of DE, it has been challenging to shift from these observations towards a coherent understanding

25 of the underlying generative processes that would essentially decode DE- a transition which is essential for progress in basic and disease biology. We address this gap by exploiting novel computational and systems biology approaches to develop a predictive model of DE based on genome-wide regulatory interaction data. Utilizing diverse genomic datasets, we identify a complex, yet strikingly consistent set of principles that control DE. This model of differential expression, called DEcode, can be applied to the majority of current and future gene expression data, to accelerate basic and disease biology, by identifying the origins of DE in each experiment.

Diverse molecular interactions have been shown to generate DE, and jointly regulate gene expression at the transcriptional and post-transcriptional levels. Major classes of gene regulatory interactions have been cataloged at the genomic scale, including transcription factor (TF)-promoter interactions ${ }^{2}$, protein-RNA interactions $^{3}$, RNA-RNA interactions ${ }^{4}$, chromatin interactions ${ }^{5}$, and epigenetic modifications on DNAs ${ }^{6}$, histones, and RNAs ${ }^{7}$. Statistical models of gene expression can help fulfill the purpose of these resources in describing the origins of gene regulation and $\mathrm{DE}^{1}$. However, such raw data resources have outpaced model development, likely due to the challenge of uniting diverse molecular data into a single accurate model.

Predicting DE on the basis of gene regulatory interactions is one initial approach to understanding its origins. Among many possible statistical approaches to predicting DE, deep learning (DL) blends diverse data sources in a way that approximates the convergence of regulatory interactions. Indeed, DL has been applied to genomic research ${ }^{8,9}$ including RNA splicing ${ }^{10}$, genomic variant functions ${ }^{11}$, and RNA/DNA binding ${ }^{12}$. However, accurate prediction is only one component of understanding DE; additional genomic and systems biology analysis are helpful in understanding how predictions are fueled by existing molecular concepts, mechanisms, and classes.

44 To decode the basis of DE in terms of molecular regulatory interactions, we first learn to predict it with a high degree of accuracy, using a DL model we call "DEcode". This model combines several types of gene regulatory interactions and allows us to prioritize the main systems and molecules that influence DE on a tissue-specific basis. We further establish

47 likely molecular mechanisms for this gene regulation and validate the influence of the predicted strongest regulators. In 48 parallel, we predict the origin of person-to-person DE, which is the major component of experimental and clinical studies. 49 These particularly challenging predictions are validated on a genome-wide scale, as we identify key drivers of coexpression, and also drivers for phenotype-associated differential expression. These tests and applications indicate

51 DEcode can combine multiple recent data sources, to extract regulators for arbitrary human DE signatures. 


\section{Results}

\section{Promoter and RNA features predict differential expression across human tissues}

54 The overarching goal of this study is to accurately predict gene expression as a function of molecular interactions. These

55 results should be tissue-specific, but also highlight major regulatory principles across tissues, and ideally have sufficient accuracy to predict the relatively small expression changes observed between individual humans. To accomplish this, we utilized deep convolutional neural networks in a system called DEcode that can predict inter-tissue variations and interperson variations in gene expression levels from promoter and mRNA features (Figure 1). The promoter features included: the genomic locations of binding sites of 762 TFs and the mRNA features encompassed the locations of binding sites of 171 RNA-binding proteins (RNABPs) and 213 miRNAs in each mRNA (Table S1). DEcode takes the promoter features and the mRNA features for each gene as inputs and outputs its expression levels under various conditions. We note that the prediction is based on only the presence or absence of known binding sites, and other information such as gene expression levels of TFs, RNABPs, and miRNAs is not utilized. First, we applied the DEcode framework to tissuespecific human transcriptomes of 27,428 genes and 79,647 transcripts measured in the GTEX consortium ${ }^{13}$ to predict log-fold changes across 53 tissues against the median log-TPM (transcripts per million) of all tissues, as well as the median log-TPM of all tissues with a multi-task learning architecture. To ensure rigorous model testing, we excluded all genes or transcripts coded in chromosome 1 from the training data and used them as the testing data for evaluating the performances of DEcode models. This procedure prevents information leaking from intra-chromosomal interactions and potential overlaps of regulatory regions (details of model construction in Figure S1). The predicted median TPM levels showed high consistency with the actual observations for both gene-level (Spearman's rho $=0.81$ ) and transcript-level

71 (Spearman's rho $=0.62)($ Figure 2A). Moreover, the model predicted the differential transcript usage within the same gene $($ Spearman's rho $=0.44)($ Figure 2A). The DEcode models also predicted the differential expression profiles across

7353 tissues for both gene (mean Spearman's rho $=0.34)$, transcript (mean Spearman's rho $=0.32$ ), and transcript-usage levels (mean Spearman's rho $=0.16)($ Figure 2B). The predicted gene expression for the testing genes was indeed tissuespecific, as they showed less correspondence with the expression profiles from alternate tissues (Figure 2C).

76 To provide context for the statistical performance of DEcode, we contrast it to a high-performing method called 77 ExPecto $^{11}$, as it was designed to predict GTEX gene expression from epigenetic states, estimated from promoter 78 sequences via DL. We built 10 models for each method using the same genes for training, validation, and testing to 
predict gene expression in the 53 tissues. In this comparison, DEcode showed an average of $7.2 \%$ improvement in root mean square error over ExPecto (Figure 2D) which translates into an average correlation coefficient with actual gene expression of 0.42 - a 50\% increase over 0.28 from ExPecto (Figure S2).

Beyond the predictive performance of DEcode, we utilize the model to help define the biological processes regulating of promoter and thereby define gene expression levels ${ }^{2}$. However, it is unclear to what extent RNA features, which we define as each RNA's binding sites of proteins and miRNA's, contribute to gene expression levels compared to TFspromoter interactions. To answer this question, we re-trained the deep learning model, randomizing either RNA features, promoter features, or both. We found that RNA features alone explained the actual TPM values better than the model trained with promoter features (Figure 2E). An example of how RNA features may distinguish between transcripts to a greater extent than promotor features can be seen in the structure of the gene ACADM (Figure 2F), which showed substantial differences between the promoter-based model and the RNA-based model. For instance, the promoter-based model could not distinguish 8 out of 11 transcripts coding for the ACADM gene that shared the same promoter region (p1 in Figure 2F). However, the actual expression levels for the 8 transcripts varied depending on the mRNA structures and therefore were more accurately captured by the RNA-based model (Figure 2F). However, the importance of RNA features was tissue-dependent (Figure 2G), as gene expression in the aorta and coronary arteries were mainly defined by TF-promoter interactions, whereas RNA-binding features were the major predictors for thyroid-specific or skeletal muscle-specific expression.

\section{Regulatory factors for differential expression across human tissues}

99 To quantify the importance of the biological interactions weighted in the DEcode models, we calculated DeepLIFT scores, which are a measure of the additive contribution of its binding site to each prediction ${ }^{14,15}$ and then averaged the DeepLIFT scores for each interactor across genes (Table S2). Because DeepLIFT scores for the gene-based model and the transcript-based model were well correlated (Spearman's rho $=0.52, \mathrm{P}<2.2 \mathrm{e}-16$ ) (Figure S3), we focused on DeepLIFT scores for the gene-based model in the following analyses. For the prediction of median TPM levels, the enrichment of the binding sites of RNABPs peaked among the top $12 \%$ of influential predictors, which was significantly greater than the influence of TFs and miRNAs $(\mathrm{P}<0.00001)$ (Figure 3A). Indeed, out of the top 30 key predictors, 19 were RNABP's binding sites and 11 were TF binding sites. The direction of DeepLIFT scores indicates either a positive 
or a negative effect of having the binding site on the abundance of RNA (Figure 3B). For instance, the binding sites of ATXN2, DDX3X, and FUS had high positive DeepLIFT scores to the prediction of RNA abundance, indicating the RNAs that bear binding sites for these RNABPs tended to be more highly expressed (Figure 3B). We also calculated DeepLIFT scores for tissue-specific expression to examine critical predictors for each of 53 tissues (Figure 3C). The DeepLIFT scores across tissues recapitulated the contribution of binding sites of known master regulators in each tissue such as REST for brain tissues ${ }^{16}$, SPI1 and RUNX1 for immune-related tissues ${ }^{17}$, TP63 and KLF4 for skin ${ }^{18}$, HNF4A

113 for liver ${ }^{19}$, and PPARG for adipose-related tissues ${ }^{20}$, which suggested the differences in predictive contributions of 114 binding sites of a given regulator reflect the differential activities of regulators across tissues. We hypothesized that the differential activities of a regulator could be in part explained by the relative abundance of a regulator across tissues. Based on this hypothesis, we contrasted DeepLIFT scores for the binding sites of each regulatory factor and its expression levels across tissues. We indeed found that 99 RNABPs and 410 TFs showed significant correlations between DeepLIFT scores of their binding sites and their expression levels (FDR $<5 \%$ ) (Figure S4). These relationships were not based on differences in expression profiles between brain and non-brain tissues, as the relationships remained the same without brain tissues (Figure S5). The sign of the correlation possibly reflects whether the binding of a regulator to RNA increased or decreased the abundance of the RNA. For instance, the model suggested that PPARG and PTBP1 are positive

regulators of gene expression as DeepLIFT scores of PPARG or PTBP1 binding sites were higher in the tissues expressing PPARG or PTBP1 at higher levels (Figure 3D). Indeed, PPARG is a transcriptional activator ${ }^{20}$ and PTBP1 is a stabilizer

of $\mathrm{RNAs}^{21}$. Conversely, the expression levels of REST, a transcriptional repressor ${ }^{16}$, or METTL14, an RNA methyltransferase destabilizing RNAs ${ }^{22}$, showed inverse correlations with their DeepLIFT scores as expected (Figure

3D). These results indicated that DEcode reflects biological mechanisms for controlling RNA abundance.

\section{Critical predictors of transcriptome are enriched for disease genes.}

Next, we characterized the roles of the critical regulators of human transcriptome, as suggested by the DEcode models

(Figure 3A). We hypothesized that if these are truly impactful transcriptome regulators, then defects in such regulators would have significant impacts on cellular phenotypes and thereby lead to disease. To examine this hypothesis, first, we obtained genes whose loss-of-function (LoF) mutations are depleted through the process of natural selection, from the Exome Aggregation Consortium (ExAC) ${ }^{23}$. Since these genes are intolerant to LoF mutations they are considered to play important roles in individual fitness. Out of all TFs and RNABPs used in DEcode, 853 genes were examined in the ExAC 
study and 601 genes were reported as being intolerant of homozygous or heterozygous LoF mutations, with probability greater than $99 \%$. We found that these LoF-mutation-intolerant regulators had greater DeepLIFT score magnitudes for the prediction of the absolute gene expression (Figure 3E and Table S3). In particular, these associations are based on genes that are intolerant to both heterozygous and homozygous LoF mutations (Figure S6). This suggested that having LoF mutations only in a single allele of the predicted critical regulators would cause a deleterious consequence on survival or reproduction in humans. Next, to examine whether the predicted critical regulators of transcriptome indeed cause diseases, we obtained disease-causing genes registered in the Online Mendelian Inheritance in Man (OMIM) ${ }^{24}$. We confirmed that mutations in the regulators with high DeepLIFT scores tended to cause genetic disorders (Figure 3E).

Interestingly, their roles on fitness are likely preserved across species, as dysfunctions of the predicted critical regulators also led pre-weaning lethality in mice (Figure 3E). Lastly, we asked whether the loss-of-function of the predicted critical regulators of the transcriptome could also impair cellular viability, by overlapping them with loss-of-function screens for a range of cellular models, from the Cancer Dependency Map project (DepMap) ${ }^{31}$. We found that the key genes for cellular viability tended to have higher DeepLIFT scores in the DEcode model (Figure 3E). These results were robust, as they were also supported by the DeepLIFT scores for the transcript-level model (Figure S7). Together, the results indicated that the critical predictors of transcriptome indeed play critical roles in maintaining vital cellular and body

\section{DEcode predicts differential expression across individuals}

154 Next, we asked whether the same input of promoter and RNA features could also predict relative expression differences across individuals within the same tissue. We hypothesized that each individual has different activation levels of regulatory factors, and thus those differences lead to person-specific differential expression of their targets. To verify our hypothesis, we extended the DEcode framework to model differential expression across individuals for 14 representative tissues with a sample size greater than 100 in GTEX. This was challenging as the average variance in gene expression within tissues was less than $25 \%$ of that between tissues (Figure S8).

To generate person-specific predictions, we utilized transfer learning, wherein the parameters in convolutional layers in the across-tissue DEcode model were fixed and then only the parameters in the fully-connected layers were tuned (Figure S9). The person-specific models successfully predicted fold changes across individuals with a mean Spearman's 
correlation of $\sim 0.28$ (Figure 4A). The performance was further increased to 0.34 when we filtered out the models that worked poorly for the validation data (Figure S10). Note the model selection was performed based on validation data alone, and all the follow-up performance evaluations and analyses were conducted by using testing data to prevent information leaks that could inflate model performance (Figure S9). The models were indeed person-specific as they did not predict gene expression profiles of unrelated individuals (Figure 4A). To examine if the model captured the personspecific expression shared across tissues ${ }^{25}$, we compared expression between tissues within the same individuals and between different individuals. The predicted expression showed better concordance between tissues from the same individuals, as is the case with actual expression data, which indicated the model captured the person-specific regulatory mechanisms, even though we did not use any direct information that could identify individuals (Figure 4B).

Next, to gauge the contribution of RNA and promoter features to the person-specific expression profiles, we re-trained models with randomized RNA features, promoter features, or both. The RNA-feature-based model performed on average $85 \%$ as well as the model trained with all features. This corresponded to an average $173 \%$ performance gain, compared to the promoter-feature-based model, which suggested that the post-transcriptional controls are the major determinants of the differential expression across individuals (Figure 4C). The model also allowed us to investigate the person-specific activities of regulators by calculating DeepLIFT scores (Figure 4D). At least 100 of regulators out of 933 regulators in each tissue showed a good correlation between their DeepLIFT scores and expression levels across individuals (Figure S11). The signs of these correlations were consistent between tissues, and consistent with those of the cross-tissue model

(Figure S12). This suggested that differential expression between individuals and between tissues can be modeled by the universal relationships between regulators and their targets.

To examine whether specific genes contributed to the per-person accuracy of the predicted gene expression, we also assessed its accuracy on a per-gene basis. The predicted expression of a majority of the testing genes ( $78 \%$ on average) showed significant positive correlations with the actual gene expression $(\mathrm{FDR}<5 \%)$. In order to assess whether this predictive performance outperformed a state of the art method, we compared DEcode with PrediXcan ${ }^{26}$, which predicts person-specific gene expression from genetic variations in cis-regulatory regions of genes. We built PrediXcan models for each of the testing genes based on the same GTEX gene expression data used for the DEcode models and wholegenome sequence data of corresponding individuals (see Methods). The PrediXcan model predicted gene expression 
This suggested that the differential activity of transcriptional and post-transcriptional regulators has a larger effect on gene expression than genetic variations in cis-regulatory regions.

The genes that DEcode could predict well were similar across tissues (Figure S13). This suggested that the predictability of gene expression is defined by gene characteristics rather than a target tissue. We, therefore, explored gene characteristics that were associated with the per-gene accuracy of the predicted expression. We found that the models showed higher performance for the genes that are registered in multiple gene annotation databases than those found only in the GENCODE database (Figure S14). The GENCODE-specific genes are novel or putative and thus their annotations are not well established. Since both actual gene expression and binding features in RNA and promoter regions are likely to be less accurate for such a novel or putative gene, it is reasonable that the performance of the model for those genes was lower than other well-established genes. Beyond the annotation reliability, we found that the number of known binding features for each gene had a larger effect on the predictability (Figure S15). This suggested that the more information on RNA and promoter interactions is available, the more the prediction becomes accurate. Interestingly, the accuracy for a portion of genes.

\section{DEcode predicts trait-related transcriptomic changes}

Next, we asked whether the person-specific expression profiles predicted by the DEcode models also retained traitassociated differential expression changes. For this, we conducted differential expression analysis against the donor's age and sex using the predicted gene expression data. Notably, test statistics of the predicted data showed significant positive correlations with those of the actual data in all tissues for both traits (Figure 5A). Especially, age- and sexspecific expression changes were well preserved in the predicted data in lung (Spearman's rho $=0.59, \mathrm{P}<2.2 \mathrm{e}-16$ ) and hippocampus (Spearman's rho $=0.47, \mathrm{P}<2.2 \mathrm{e}-16$ ), respectively. The predicted associations were the closest to those of corresponding tissues in 9 and 11 out of 14 tissues for age and sex, respectively (Figure 5B). This indicated that the predicted gene expression changes against age and sex are tissue-specific in most cases, rather than the effects shared across tissues. We also explored the regulators for the age- and sex-related gene expression changes by associating regulator's DeepLIFT scores with age and sex. We found that many regulators, for instance, 717 in the tibial artery and 
218904 in the breast mammary tissue, showed age- and sex-dependent changes at FDR 5\%, respectively (Figure 5C and

219 Table S4), which showed the capability of DEcode to associate transcriptional regulators with phenotypes. Although 220 there were more TFs associated with phenotypes than RNABPs and miRNAs, overall collective impacts of RNA features 221 on the generative process of DEs for age and sex were greater than those of promoter features in most tissues (Figure 222 S16).

\section{DEcode predicts gene co-expression relationships}

Co-expression analysis is a frequent component of transcriptome studies as gene-to-gene co-expression relationships are regarded as functional units of the transcriptional system ${ }^{27}$. Therefore, we examined if the DEcode models could detect known gene co-expression relationships. These tests were both a potential validation of the person-specific DEcode predictions, and a means to explore the biological basis of co-expression. We found that the gene co-expression relationships in the predicted gene expression profiles separated gene pairs with positive and negative correlation in the actual gene expression data in each tissue (Figure 6A). Furthermore, the predicted gene expression profiles also detected inter-tissue co-expression relationships (Figure 6B). The accuracy of these results motivated us to investigate key factors driving co-expression, via the DEcode predictions. RNA features alone could explain co-expression relationships better than promoter features in most tissues (Figure 6C), which again suggested the significant contribution of RNA features to person-specific transcriptomes.

To further assess the capability of DEcode to decipher the mechanisms leading a specific co-expression relationship, we focused on the co-expression of LAPTM5 and CD53, which were robustly co-expressed both in the simulated expression data and the actual data in all tissues except whole-blood. Using the trained model, we simulated the consequences of disruptions of promoter and mRNA features. The co-expression relationship was weakened when the features near transcriptional start site (TSS) and 1,000 bp downstream of TSS in LAPTM5 or near TSS and $500 \mathrm{bp}$ upstream of TSS in CD53 were removed (Figure 6D). These observed effects were reasonable because many TFs bind to these regions (Figure 6D). We further examined the specific regulators for the co-expression relationships by simulating knockout (KO) effects of regulators. The in-silico KO experiments revealed that immune-related TFs such as SPII and TBX21 potentiated the co-expression relationships consistently across multiple tissues (Figure 6E). To validate if these regulators indeed induced the co-expression relationships, we conducted a mediation analysis that is an orthogonal computational method to infer the effect of regulators on downstream targets. A mediation analysis evaluated the 
246 hypothesis where if LAPTM5 and CD53 are co-expressed due to the predicted regulators, normalizing expression levels

247 of the two genes by the expression levels of the regulators would decrease the co-expression relationships. Specifically,

248 it quantified the covariance between LAPTM5 and CD53 explained by the expression levels of the predicted regulators

249 using the actual expression data. The set of the 10 regulators together mediated up to $94 \%$ of covariance, which was

250 significantly greater than the same number of randomly picked regulators (Figure 6F). This example showed the utility

251 of DEcode framework to identify the drivers of the co-expression.

DEcode reveals molecular regulations for frequently DE genes in meta-transcriptomes

254 A recent meta-analysis of over 600 human transcriptome data revealed that some genes are more likely to be detected as

DE genes than others in diverse case-control studies ${ }^{28}$. From this observation, Megan et al. formulated the "DE prior", a global ranking of gene's generic likelihood of being DE. The genes with high DE prior rank were significantly more enriched with DE genes from a variety of conditions, as compared to other functional gene sets, such as those contained in gene ontology or canonical pathways ${ }^{28}$. However, the regulatory-origin behind the ranking of these highly responsive genes has yet to be uncovered. Therefore, we used DEcode to examine whether the DE prior rank could be generated by gene regulatory interactions, and to identify critical regulatory relationships for frequently DE genes. The ability of DEcode to predict global DE prior ranks was highly significant $(\mathrm{P}<2.2 \mathrm{e}-16)$ and practically relevant (Spearman's rho $=$ 0.53) (Figure 7A). Furthermore, DEcode was able to identify genes with high (90th percentile and greater) DE prior probability $($ AUCROC $=0.81,95 \%$ confidence interval $=0.78-0.84)($ Figure 7 B $)$. Re-training the model with randomized inputs indicated that TF-promoter interactions were the major factors explaining the DE prior rank (Figure 7B). To further characterize TFs that contributed to the prediction, we defined TFs with DeepLIFT score greater than 90th percentile as critical TFs (Table S5) and performed pathway analysis on them. We found that critical TFs were enriched for cancer or inflammatory-related KEGG pathways $(\mathrm{FDR}<5 \%)$ such as pathways in cancer (Fold $=3.1, \mathrm{P}=$ 4.2e-5), JAK-STAT signaling pathway (Fold $=6.8, \mathrm{P}=4.8 \mathrm{e}-5)$, chemokine signaling pathway $($ Fold $=7.3, \mathrm{P}=1.4 \mathrm{e}-4)$, and acute myeloid leukemia (Fold $=4.5, \mathrm{P}=3.6 \mathrm{e}-4$ ) (Table S6). This result is consistent with the disease-related data context for DE prior, which is $62 \%$ cancer-related and $23 \%$ inflammatory-related. Supported by the ability to predict DE lists, to uncover major key drivers for generating DE. 
273 In summary, DEcode defines major principles in gene regulation in arbitrary gene expression data. It is applicable to

274 tracing the origins of complex gene expression patterns such as co-regulation, and also to arbitrary gene expression

275 signatures. This capacity is strongly supported on a comparative basis to alternative methods, and on an absolute basis

276 across diverse applications, which include, through predictions of transcript-usage, person-specific gene expression,

277 frequently DE genes of multiple external disease-related gene sets.

\section{Discussion}

279 We introduced the DEcode framework, which integrates a wealth of genomic data into a unified computational model of 280 transcriptome regulations to predict multiple transcriptional effects, including the absolute expression differences across 281 genes and transcripts, tissue- and person-specific transcriptomes. Systems biology analysis of these results provided 282 biological insights regarding the regulatory mechanisms of transcriptome. For instance, it suggested that absolute 283 expression levels are mainly under post-transcriptional control, whereas tissue-specific expression is shaped by both 284 transcriptional and post-transcriptional control. This implied that TFs act as a switch that initiates tissue-specific 285 transcriptional programs, but once a gene is transcribed at a certain level, its abundance in the cells will be primarily 286 regulated by RNABPs. The post-transcriptional regulators were also critical for explaining individual differences in 287 transcriptomes and thus may fine-tune the transcriptome in response to environmental and genetic factors.

288 Transcriptome analysis often identifies differentially expressed genes and then assesses the enrichment of functional 289 genes such as TF-targets one by one. The person-specific DEcode model offers several comparative advantages. First, 290 DEcode can take into account the effects of multiple regulators simultaneously as opposed to one at a time. Second, 291 DEcode can estimate the person-specific regulator's activities that can be used to identify regulators associated with a 292 phenotype of interest. Third, DEcode can simulate the consequence of KO perturbations for each gene. This step can 293 reduce the number of candidate key drivers of gene expression changes by an order of magnitude or more, and facilitates 294 the design of follow-up experiments. Therefore, DEcode can extract more actionable information from transcriptome 295 data, which will benefit a variety of transcriptome studies.

296 Looking toward even more expansive applications, the DEcode framework has the flexibility to incorporate other types 297 of genomic information such as DNA methylation, histone marks, and RNA modifications, and also can be extended to 298 other organisms. Thus, DEcode framework provides a direct bridge between accumulating genomic big data and 
299 individual transcriptome studies, allowing researchers to predict molecules that control DE associated with any condition 300 or disease.

\section{Materials and Methods}

Transcriptome data processing

303 To prepare gene expression data used for the model training, we downloaded the median gene TPM from 53 human 304 tissues from the v7 release of GTEX portal (https://gtexportal.org). We kept 27,428 genes expressed greater than two 305 TPM in at least one tissue and $\log 2$-transformed TPM with the addition of 0.25 to avoid a negative infinity. Then, we 306 calculated the median $\log 2$-TPM across 53 tissues and $\log 2$-fold-changes relative to the median of all tissues. The 307 processed gene-level expression data comprised 27,428 genes with 54 columns including relative fold-changes for 53 308 tissues and the median $\log 2$-TPM across 53 tissues. To compile transcript-level data, we downloaded the individual309 level transcript TPM from the GTEX portal and computed the median transcript TPM by tissue. We processed the 310 transcript data in the same way we did for the gene-level data. The resulted transcript-level data included 79,647

311 transcripts that corresponded to 23,813 genes. For building person-specific DEcode models, we obtained the gene-level

312 TPM for each individual in 14 tissues from the GTEX portal. We filtered out lowly-expressed genes in each tissue and 313 kept genes expressed greater than one TPM in at least 50\% of samples. Then, we log2-transformed TPM with the 314 addition of 0.25 and then quantile normalized the $\log 2-$ TPM. Finally, we removed the effects of technical covariates 315 including rRNA rate, intronic rate, and RIN number via linear regression for each gene followed by quantile 316 normalization.

317 Promoter and RNA binding features.

318 To generate RNA and DNA feature matrices, we downloaded genomic locations of binding sites of 171 RNABPs from 319 POSTAR2 $^{29}$ as of Oct 2018, 218 miRNAs from TargetScan Release 7.2 $2^{30}$, and 826 TFs from GTRD ${ }^{31}$ as of Oct 2018. 320 Then, we mapped the binding sites of RNABPs, miRNAs, and TFs to promoters and RNA-coding regions defined in 321 the GTF file provided by the GTEX portal. A promoter region of each gene was defined as the region from 2,000 bp 322 upstream of the transcriptional start site (TSS) to 1,000bp downstream of the TSS. We only used interactors that bind to 
promoters or RNA-coding regions of at least 30 genes, or transcripts as the predictors in each model. To reduce the size

324 of the input, an RNA-coding region and a promoter region of each gene was binned with $100 \mathrm{bp}$ intervals and the number of bases bound to each RNABP, miRNA, or TF was counted in each interval. This step generated RNA and DNA feature matrices for each gene described in Figure 1.

\section{Training tissue-specific models}

328 For training the gene-level model of tissue-specific expression, we reserved all 2,705 genes coded on chromosome 1 as the testing data and the rest of the genes was randomly split into training data $(22,251$ genes $)$ and validation data $(2,472$ genes). In the case of the transcript model, we used all 7,631 transcripts coded on chromosome 1 as the testing data and the rest of the transcripts was randomly split into training data (64,978 transcripts) and validation data $(7,038$ transcripts). The binding matrices were normalized by the maximum values for each binding protein and miRNA. The relative fold-changes for 53 tissues were scaled together to set the standard deviation as one and the median log2-TPM was separately scaled to set the standard deviation as one. These steps were conducted for the training data first and then the same scaling factors were used for the validation and the testing data to avoid information leaking from those data. We constructed and trained DL models using Keras (version 2.1.3) $)^{32}$ with a TensorFlow (version 1.4.1) $)^{33}$ backend. Hyper-parameters were optimized using hyperopt (version 0.2$)^{34}$ based on the mean squared error against the validation data. The detailed structure of the model was described in Figure S17. The training was done using minibatches of 128 training examples with a learning rate of 0.001 for Adam optimizer ${ }^{35}$. The number of maximum training epochs was set to 100 with early-stopping of 10 based on validation loss. This training cycle was repeated 10 times and the best model for the validation data was selected as the final model (Figure S1). All models were trained using TITAN X Pascal graphics processing units (Nvidia).

\section{Comparison of DEcode with ExPecto}

344 To perform a fair comparison between DEcode and ExPecto ${ }^{11}$, we used 18,550 genes that were commonly included in 345 both studies and trained models with the same set of genes for training and evaluation. Since ExPecto model was 346 originally built using genes on chromosome 8 as the testing data, we followed the same procedure as we reserved all 347714 genes coded on chromosome 8 as the testing data and the rest of the genes was randomly split into training data 348 (16,052 genes) and validation data (1784 genes). The epigenetic states estimated by ExPecto were downloaded from 
the ExPecto repository (https://github.com/FunctionLab/ExPecto) as of Nov 2019. Given the epigenetic states, we built

a prediction model for tissue-specific gene expression for each tissue via XGBoost based on the training script

downloaded from the ExPecto repository. We modified the original script so that the early stopping of the model

optimization was decided based on the performance on the validation data instead of the testing data. This modification prevented the overfitting of the model to the testing data. We used the same hyper-parameters for XGBoost as in the script. Both hyper-parameters and model parameters of DEcode model were also trained with the same set of training, validation, and testing genes.

\section{6}

\section{DeepLIFT score calculation}

To evaluate the importance of input features to the prediction, we calculated DeepLIFT (Deep SHAP) scores ${ }^{14}$ using DeepExplainer implementation (version 0.27 .0$)^{15}$. The DeepLIFT method estimates the contribution of each input compared to a reference input in a trained DL model. To compute the contribution of the presence of a binding site, we used a reference that does not have any binding sites in both promoters and RNAs with the median length of all genes in the testing data. DeepLIFT scores follow a summation-to-delta property where the summation of input contributions (DeepLIFT scores) is equal to the difference in the predicted value compared to the prediction from the reference input. We calculated DeepLIFT scores for each gene in testing data for each of 54 outputs, then summed up the scores over promoter or RNA regions for each feature, and finally averaged them over genes.

\section{Disease genes}

The probability that a gene is intolerant for a loss-of-function mutation was downloaded from the release 1.0 of the ExAC portal (http://exac.broadinstitute.org). Disease genes were obtained from the OMIM portal as of June 2019 (https://www.omim.org/). We excluded provisional gene-to-phenotype associations and genes associated with nondisease phenotypes, multifactorial disorders, or infection. We obtained mouse-lethal genes from Gene Discovery Informatics Toolkit (v1.0.0) ${ }^{36}$ that provided pre-processed gene lists from the murine knock-out experiments registered in Mouse genome informatics (MGI) ${ }^{37}$ and the International Mouse Phenotyping Consortium (IMPC) ${ }^{38}$. The results of CRISPR screening for the genes essential for proliferation or viability conducted in the DepMap project ${ }^{39}$ were downloaded from Enrichr portal ${ }^{40,41}$ as of June 2019 (https://amp.pharm.mssm.edu/Enrichr). Enrichr portal provided two CRISPR screening results conducted independently at Broad Institute and the Sanger Institute. To reduce the false 
375 positives in the CRISPR screening, we used essential genes that were identified in both of the two independent

376 screenings.

\section{Training person-specific models}

378 To train person-specific models, we utilized the same model structure as the tissue-model, except that the number of 379 model outputs was modified to match the sample size of the tissue. We re-used the parameters of convolutional layers 380 in the tissue-model and only parameters in the fully-connected layers were tuned (Figure S9). We used the same gene 381 splits and the same procedure of normalization and scaling as the tissue-model for training and evaluating models. We 382 evaluated the model prediction for each individual separately based on validation data and filtered out the individual 383 models that performed less than 50\% percentile of all individual models for some analyses (Figure S9).

\section{Training PrediXcan models}

385 To build a prediction model for gene expression from genotype data, we trained PrediXcan ${ }^{26}$ models with GTEX gene 386 expression and genotype data. A QCed vcf file of GTEx genotype data called by whole-genome sequence was 387 downloaded from dbGaP for 635 individuals. We filtered out variants with a missing rate greater than $1 \%$ and minor 388 allele frequency less than 1\% and kept 9,219,660 variants for PrediXcan. We followed the model building procedure 389 employed in PredictDB (http://predictdb.org/), a repository of PrediXcan models, as of Nov 2019. Briefly, we 390 randomly split the samples into 5 folds. Then for each fold, we removed the fold from the data and used the remaining 391 data to train an elastic-net model using 10-fold cross-validation to tune the lambda parameter. With the trained model, 392 we predicted gene expression values for the hold out samples. We applied the PrediXcan method to predict the same 393 gene expression data used for the person-specific DEcode models. We built PrediXcan model for each gene using

394 variants located within $1 \mathrm{Mbp}$ upstream and downstream of its TSS. A missing value of the genotype data was replaced 395 with an average dosage of non-missing samples.

\section{Differential expression analysis for age and sex}

397 Limma ${ }^{42}$ was used to identify genes associated with age using gender as a covariate. The log2-TPM values of genes in 398 the testing data were used. We also tested the associations between DeepLIFT scores for predictors and age via limma 
to identify regulators for DE against ages and sex. The Benjamini-Hochberg procedure was used to control the false discovery rate at $5 \%$.

To simulate the consequence of the removal of binding sites on the expression of LAPTM5 and CD53, we generated 10,000 synthetic inputs for each of LAPTM5 and CD53 where all binding sites in each interval of its promoter and RNA were randomly removed. From each of these synthetic inputs, we computed predicted expression values and correlated them with ones of another gene without any disruptions in its binding sites. Then, we used multiple linear regression to associate the location of disrupted regions with the correlation values between LAPTM5 and CD53 to

409 To simulate the effect of regulator knockout (KO) on the expression of LAPTM5 and CD53, we generated 10,000

410 synthetic inputs for each LAPTM5 and CD53 where each protein or miRNA bound to its promoter or RNA was

411 randomly removed from it feature matrices. From each of these synthetic inputs, we computed predicted expression

412 values and correlated them with ones of another gene without any removals in its feature matrices. Then, we used

413 multiple linear regression to associate KOs of regulators with the correlation values between LAPTM5 and CD53 to

414 estimate the effects of the KO of each regulator on the co-expression relationship. We applied the Bonferroni correction 415 to control multiple testing and the regulators with the corrected p-value less than 0.05 in all tissues were chosen as the key drivers of the co-expression.

\section{Conditional independence test}

418 To validate the effect of the predicted drivers on co-expression, we conducted a conditional independence test. We 419 regressed the actual $\log 2$-TPM values of LAPTM5 and CD53 with the actual log2-TPM values of the predicted drivers 420 and computed $\mathrm{R}^{2}$ (variance explained) between the residuals of two genes. The $\mathrm{R}^{2}$ based on the actual gene expression 421 and one from the residuals were compared to quantify the covariance explained by the predicted drivers. To evaluate 422 the significance of this effect, we repeated this process 1,000 times with an equal number of randomly picked genes 423 that have a binding site in LAPTM5 or CD53 as regressors. 


\section{DEcode model for DE prior rank}

425 DE prior rank was downloaded from https://github.com/maggiecrow/DEprior. In the DE prior rank, each gene has a

426 probability-like value where zero is the minimum and one is the maximum. To convert this value to a non-bounded

427 scale, we applied the logit transformation to the DE prior value. We assigned a value of 10 to a gene that had an infinite

428 value after the logit transformation. We used the same gene splits as the GTEX-tissue-model, which resulted in 13,433

429 genes for training, 1,504 genes for validation, and 1,674 genes for testing. We trained the DEcode model for DE prior

430 rank using the same procedure as with the GTEX-person-specific models. To evaluate the contribution of promoter and

431 RNA features to the prediction, the model was also trained with randomized input features. Receiver operating

432 characteristic (ROC) curve analysis was performed using pROC R package ${ }^{43}$ with a default setting. We performed

433 pathway analysis of the TFs with a DeepLIFT score greater than 90th percentile using KEGG pathways ${ }^{44}$. KEGG

434 pathway gene sets were downloaded from MSigDB v6.14. The enrichment significance was based on results of the

435 hypergeometric test, with 757 unique TF genes as a background, against KEGG pathways comprised of at least 5

436 background genes. FDR was controlled at 5\%. We manually curated the 159 disease-related data sets used in the

437 construction of the DE prior ranking, to determine the number of data sets related to cancer or inflammatory disease.

438 Code and model availability

439 DEcode software and pre-trained models for tissue- and person-specific transcriptomes are available at

$440 \quad$ www.differentialexpression.org.

\section{Acknowledgments}

442 We thank Dr. Lei Yu for managing access to GTEX data. The study was supported by NIH grants P30AG010161, 443 R01AG061798, and R01AG057911. The Genotype-Tissue Expression (GTEx) Project was supported by the Common 444 Fund of the Office of the Director of the National Institutes of Health, and by NCI, NHGRI, NHLBI, NIDA, NIMH, and 445 NINDS. The data used for the analyses described in this manuscript were obtained from the GTEx Portal on 10/01/2018. 


\section{Author Contributions}

447 ST contributed to the conception and design of the study. ST performed the computational analysis. ST, CG, SM, and

448 YW interpreted the result. ST wrote the first draft of the manuscript. All authors contributed to manuscript revision, read 449 and approved the submitted version.

451 1. Lee, T. I. \& Young, R. a. Transcriptional regulation and its misregulation in disease. Cell 152, 1237-51 (2013).

452 2. Lambert, S. A. et al. The Human Transcription Factors. Cell 172, 650-665 (2018).

453 3. Glisovic, T., Bachorik, J. L., Yong, J. \& Dreyfuss, G. RNA-binding proteins and post-transcriptional gene

454 regulation. FEBS letters 582, 1977-86 (2008).

455 4. Bartel, D. P. MicroRNAs: target recognition and regulatory functions. Cell 136, 215-33 (2009).

456 5. Schoenfelder, S. \& Fraser, P. Long-range enhancer-promoter contacts in gene expression control. Nature reviews.

457 Genetics 20, 437-455 (2019).

458 6. Smith, Z. D. \& Meissner, A. DNA methylation: roles in mammalian development. Nature reviews. Genetics 14, 204-

$45920(2013)$.

460 7. Roundtree, I. A., Evans, M. E., Pan, T. \& He, C. Dynamic RNA Modifications in Gene Expression Regulation. Cell $461 \quad \mathbf{1 6 9}, 1187-1200(2017)$.

462 8. Avsec, $\check{Z}$ et al. The Kipoi repository accelerates community exchange and reuse of predictive models for genomics. 463 Nature biotechnology 37, 592-600 (2019).

464 9. Libbrecht, M. W. \& Noble, W. S. Machine learning applications in genetics and genomics. Nature reviews. Genetics $46516,321-32(2015)$.

466 10. Jaganathan, K. et al. Predicting Splicing from Primary Sequence with Deep Learning. Cell 176, 535-548.e24

467 (2019).

468 11. Zhou, J. et al. Deep learning sequence-based ab initio prediction of variant effects on expression and disease risk.

$469 \quad$ Nature genetics 50, 1171-1179 (2018).

470 12. Alipanahi, B., Delong, A., Weirauch, M. T. \& Frey, B. J. Predicting the sequence specificities of DNA- and RNA- 
471 binding proteins by deep learning. Nature biotechnology 33, 831-8 (2015).

472 13. Melé, M. et al. Human genomics. The human transcriptome across tissues and individuals. Science (New York,

$473 \quad$ N.Y.) $348,660-5(2015)$.

474 14. Shrikumar, A., Greenside, P. \& Kundaje, A. Learning Important Features Through Propagating Activation

475 Differences. (2017).

476 15. Lundberg, S. M. \& Lee, S. A Unified Approach to Interpreting Model Predictions. , 4765-4774 (2017).

477 16. Chong, J. A. et al. REST: a mammalian silencer protein that restricts sodium channel gene expression to neurons.

478 Cell 80, 949-57 (1995).

479 17. Imperato, M. R., Cauchy, P., Obier, N. \& Bonifer, C. The RUNX1-PU.1 axis in the control of hematopoiesis.

480 International journal of hematology 101, 319-29 (2015).

481 18. Soares, E. \& Zhou, H. Master regulatory role of p63 in epidermal development and disease. Cellular and molecular

482 life sciences : CMLS 75, 1179-1190 (2018).

483 19. Watt, A. J., Garrison, W. D. \& Duncan, S. A. HNF4: a central regulator of hepatocyte differentiation and function.

484 Hepatology (Baltimore, Md.) 37, 1249-53 (2003).

485 20. Lefterova, M. I., Haakonsson, A. K., Lazar, M. A. \& Mandrup, S. PPAR $\gamma$ and the global map of adipogenesis and 486 beyond. Trends in endocrinology and metabolism: TEM 25, 293-302 (2014).

487 21. Ge, Z., Quek, B. L., Beemon, K. L. \& Hogg, J. R. Polypyrimidine tract binding protein 1 protects mRNAs from 488 recognition by the nonsense-mediated mRNA decay pathway. eLife 5 (2016).

489 22. Wang, Y. et al. N6-methyladenosine modification destabilizes developmental regulators in embryonic stem cells.

$490 \quad$ Nature Cell Biology 16, 191-198 (2014).

491 23. Lek, M. et al. Analysis of protein-coding genetic variation in 60,706 humans. Nature 536, 285-91 (2016).

492 24. Online Mendelian Inheritance in Man, OMIM®. McKusick-Nathans Institute of Genetic Medicine, Johns Hopkins 493 University (Baltimore, MD), \{June 11th, 2019\}. World Wide Web URL: https://omim.org/.

494 25. Ardlie, K. G. et al. The Genotype-Tissue Expression (GTEx) pilot analysis: Multitissue gene regulation in humans.

495 Science 348, 648-660 (2015).

496 26. Gamazon, E. R. et al. A gene-based association method for mapping traits using reference transcriptome data.

$497 \quad$ Nature genetics 47, 1091-8 (2015).

498 27. Gaiteri, C., Ding, Y., French, B., Tseng, G. C. \& Sibille, E. Beyond modules and hubs: the potential of gene 
coexpression networks for investigating molecular mechanisms of complex brain disorders. Genes, brain, and behavior

13, 13-24 (2014).

28. Crow, M., Lim, N., Ballouz, S., Pavlidis, P. \& Gillis, J. Predictability of human differential gene expression. Proc.

Natl. Acad. Sci. U. S. A. 116, 6491-6500 (2019).

29. Zhu, Y. et al. POSTAR2: deciphering the post-transcriptional regulatory logics. Nucleic acids research 47, D203-

D211 (2019).

30. Agarwal, V., Bell, G. W., Nam, J. \& Bartel, D. P. Predicting effective microRNA target sites in mammalian mRNAs. eLife 4 (2015).

31. Yevshin, I., Sharipov, R., Valeev, T., Kel, A. \& Kolpakov, F. GTRD: a database of transcription factor binding

sites identified by ChIP-seq experiments. Nucleic acids research 45, D61-D67 (2017).

32. Chollet, F. keras. GitHub repository https://github.com (2015).

511 34. Bergstra, J., Komer, B., Eliasmith, C., Yamins, D. \& Cox, D. D. Hyperopt: a Python library for model selection and 512 hyperparameter optimization. Computational Science \& Discovery 8, 014008 (2015).

513 35. Kingma, D. P. \&Ba, L. J. Adam: A Method for Stochastic Optimization, arXiv.org, 2015).

514 36. Dawes, R., Lek, M. \& Cooper, S. T. Gene discovery informatics toolkit defines candidate genes for unexplained 515 infertility and prenatal or infantile mortality. NPJ genomic medicine 4, 8-11 (2019).

516 37. Smith, C. L., Blake, J. A., Kadin, J. A., Richardson, J. E. \& Bult, C. J. Mouse Genome Database (MGD)-2018:

517 knowledgebase for the laboratory mouse. Nucleic Acids Res. 46, D836-D842 (2018).

518 38. Koscielny, G. et al. The International Mouse Phenotyping Consortium Web Portal, a unified point of access for 519 knockout mice and related phenotyping data. Nucleic Acids Res. 42, 802 (2014).

520 39. Tsherniak, A. et al. Defining a Cancer Dependency Map. Cell 170, 564-576.e16 (2017).

521 40. Kuleshov, M. V. et al. Enrichr: a comprehensive gene set enrichment analysis web server 2016 update. Nucleic 522 acids research 44, 90 (2016).

523 41. Chen, E. Y. et al. Enrichr: interactive and collaborative HTML5 gene list enrichment analysis tool. BMC 524 Bioinformatics 14, 128 (2013).

525 42. Ritchie, M. E. et al. limma powers differential expression analyses for RNA-sequencing and microarray studies. $526 \quad$ Nucleic acids research 43, e47 (2015). 
bioRxiv preprint doi: https://doi.org/10.1101/2020.01.10.894238; this version posted January 11,2020 . The copyright holder for this preprint (which was not certified by peer review) is the author/funder, who has granted bioRxiv a license to display the preprint in perpetuity. It is made available under aCC-BY 4.0 International license.

527 43. Robin, X. et al. pROC: an open-source package for R and S+ to analyze and compare ROC curves. BMC

Bioinformatics 12, 77 (2011).

529 44. Kanehisa, M. \& Goto, S. KEGG: kyoto encyclopedia of genes and genomes. Nucleic Acids Res. 28, 27-30 (2000).

530 45. Liberzon, A. et al. The Molecular Signatures Database (MSigDB) hallmark gene set collection. Cell systems 1, 417-

$531425(2015)$

532 Figure legends

\section{Figure 1}

\section{Model inputs}

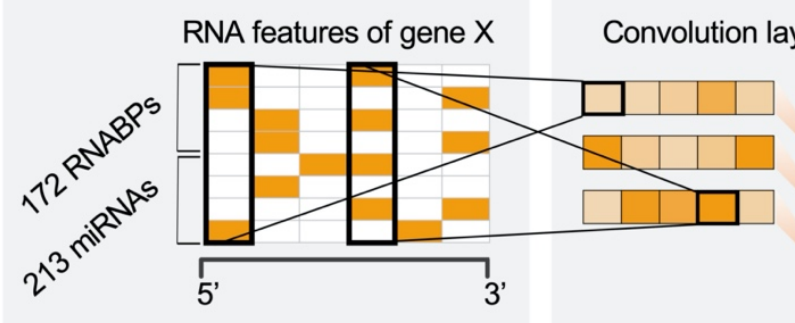

Promoter features of gene $X$

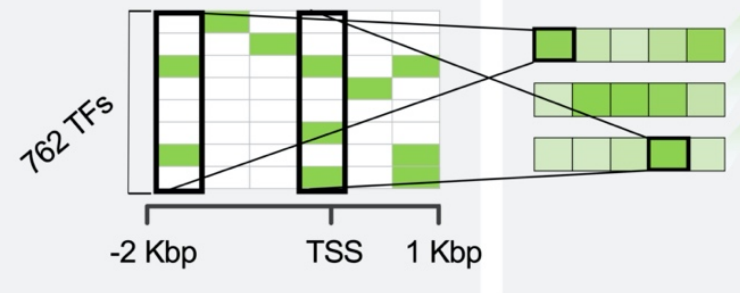

\section{Feature extraction}

Convolution layer

Fully-connected layer

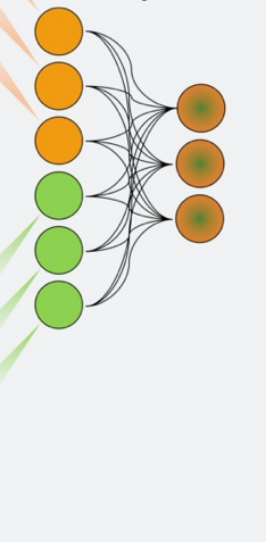

\section{Model outputs}

Gene and transcript prediction
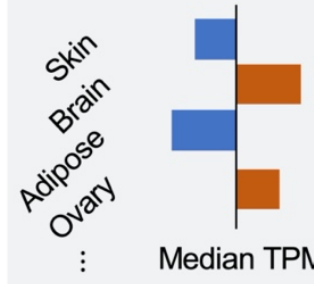

Tissue-level

Predicts RNA-seq for:

53 human tissues

27,428 genes

79,647 transcripts

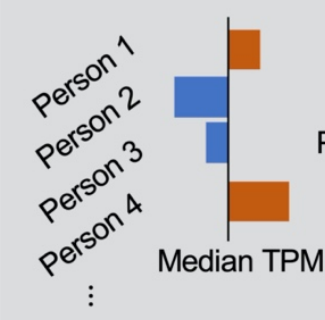

\section{Evaluation}

Accuracy by tissue

Tissuelevel
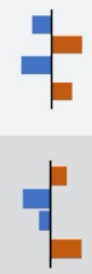

Personlevel
Transcript predictions

vs. ExPecto

Predictive performance

vs. PrediXcan

Within-tissue, person-level predictions
TF vs. RNABP vs. miRNA

Expr level of regulators

Molecular \& functional enrichment

Identity of top predictors

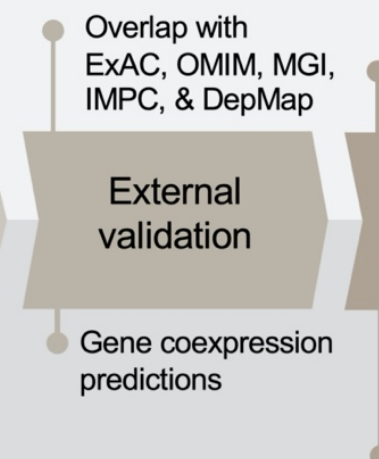

Person-specific predictions

Translational applications

DE for age and sex

Simulated coexpression Frequently DE genes

534 Figure 1. Overview of building and evaluating the DEcode transcriptome prediction model. 
bioRxiv preprint doi: https://doi.org/10.1101/2020.01.10.894238; this version posted January 11 2020. The copyright holder for this preprint (which was not certified by peer review) is the author/funder, who has granted bioRxiv a license to display the preprint in perpetuity. It is made available under aCC-BY 4.0 International license.

Figure 2
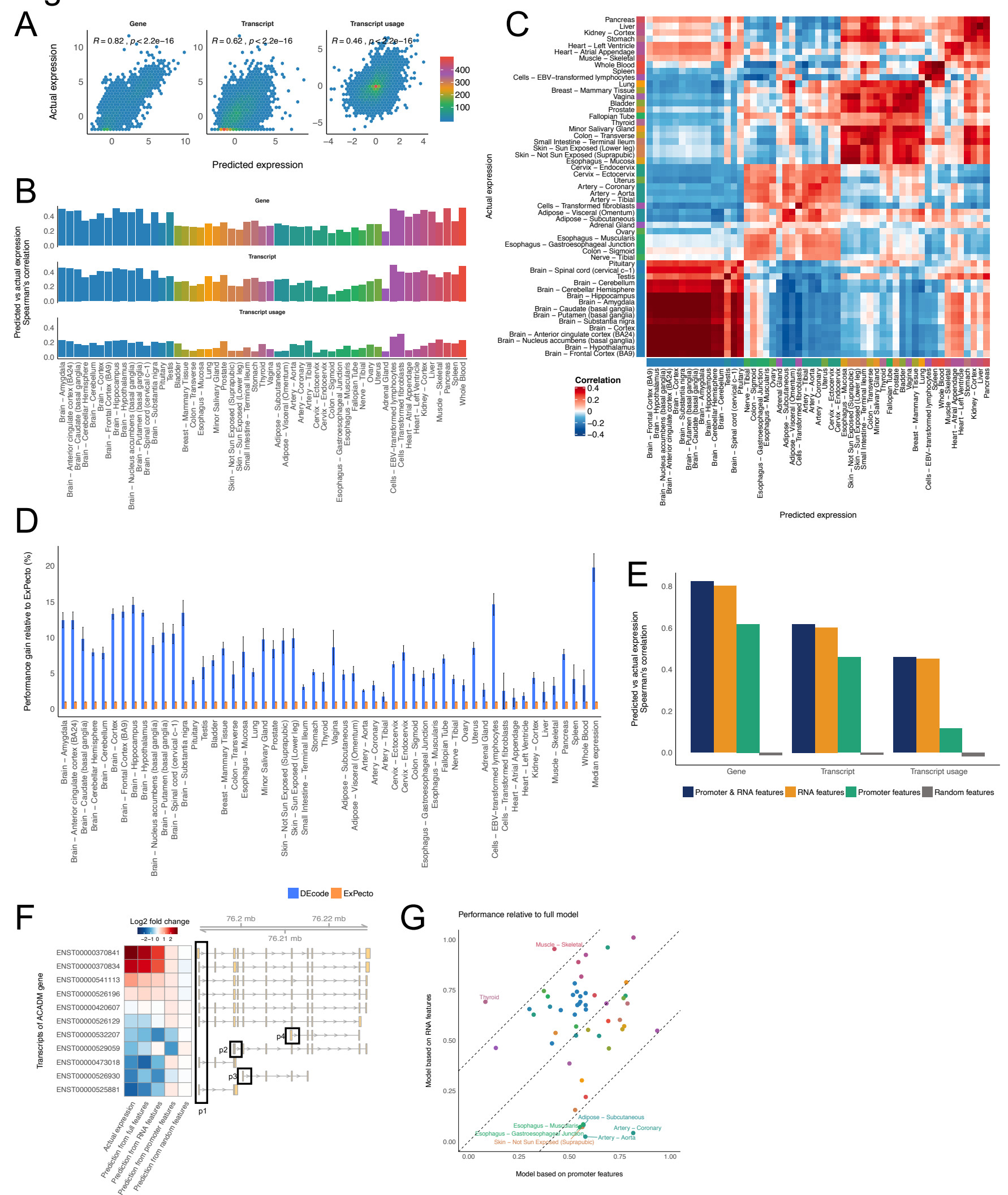

536 Figure 2. Performance of the tissue-level models. (A) Prediction performances on the median absolute expression levels 
538 compared with the actual median log2-TPMs across 53 tissues using Spearman's rank correlation. The transcript usage

539 within each gene was computed by subtracting the mean $\log 2$-TPM from log2-TPM of transcripts in each gene for 1,485

540 genes that had multiple transcripts. (B) Prediction performances on the tissue-specific expression profiles. The predicted

541 fold changes relative to the median of all tissues for 2,705 genes or 7,631 transcripts coded on chromosome 1 were

542 compared with the actual fold changes in each tissue using Spearman's rank correlation. The differences in the transcript

543 usage within each across tissues were computed for 1,485 genes that had multiple transcripts. The color of the bar

544 indicated the tissue groups based on the similarity of gene expression profiles. (C) The heatmap showing pairwise

545 correlations between the predicted and the actual tissue-specific expression profiles of 53 tissues for the testing genes.

546 (D) Performance comparison of DEcode with ExPecto. The root-mean-square errors (RMSE) of DEcode models for

547 expression-levels of 714 genes coded on chromosome 8 was compared with those of ExPecto. Each method was executed

54810 times. The median RMSE of the 10 runs was displayed as a bar plot and the error bar represents median absolute

549 deviation. (E) The predictive performances of the models trained with a different set of features. (F) The comparison of

550 the expression levels for ACADM transcripts predicted by the models trained with different feature sets. (G) The

551 predictive performances on the tissue-specific gene expression profiles of the testing data relative to the model trained

552 with a full set of features. 


\section{Figure 3}

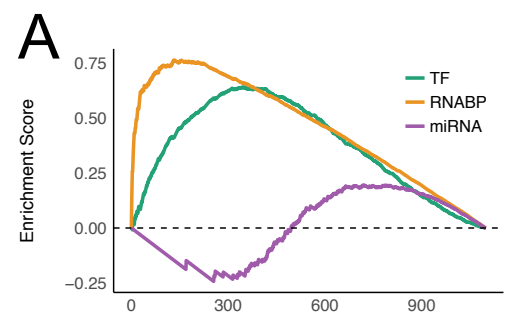

Regulator ranked by the absolute DeepLIFT score

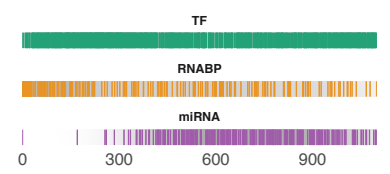

B variable importance

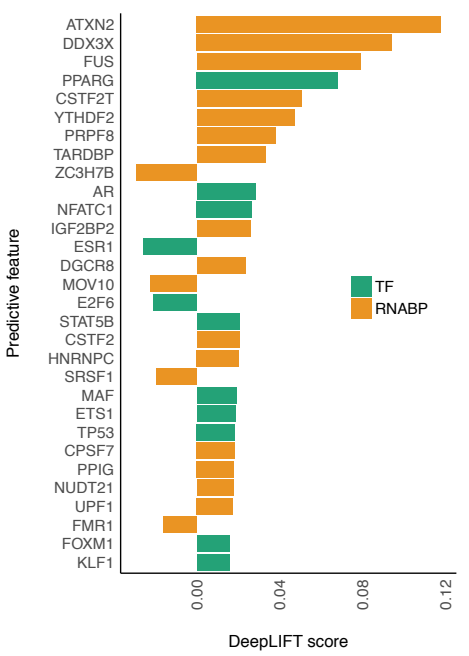

C
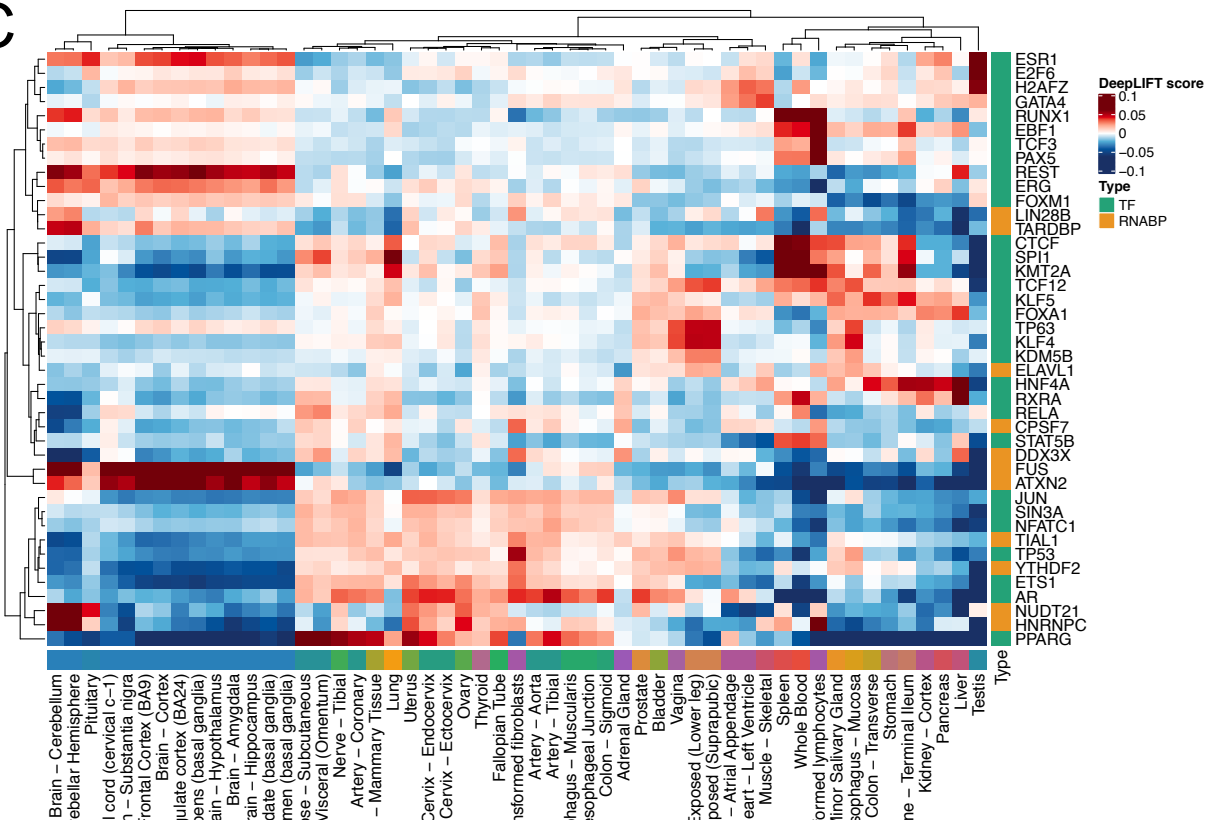

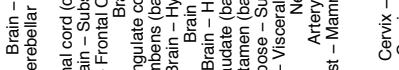
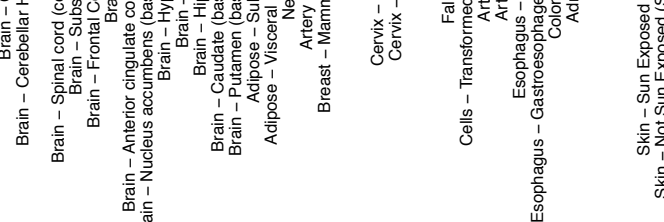

$\mathrm{E}$
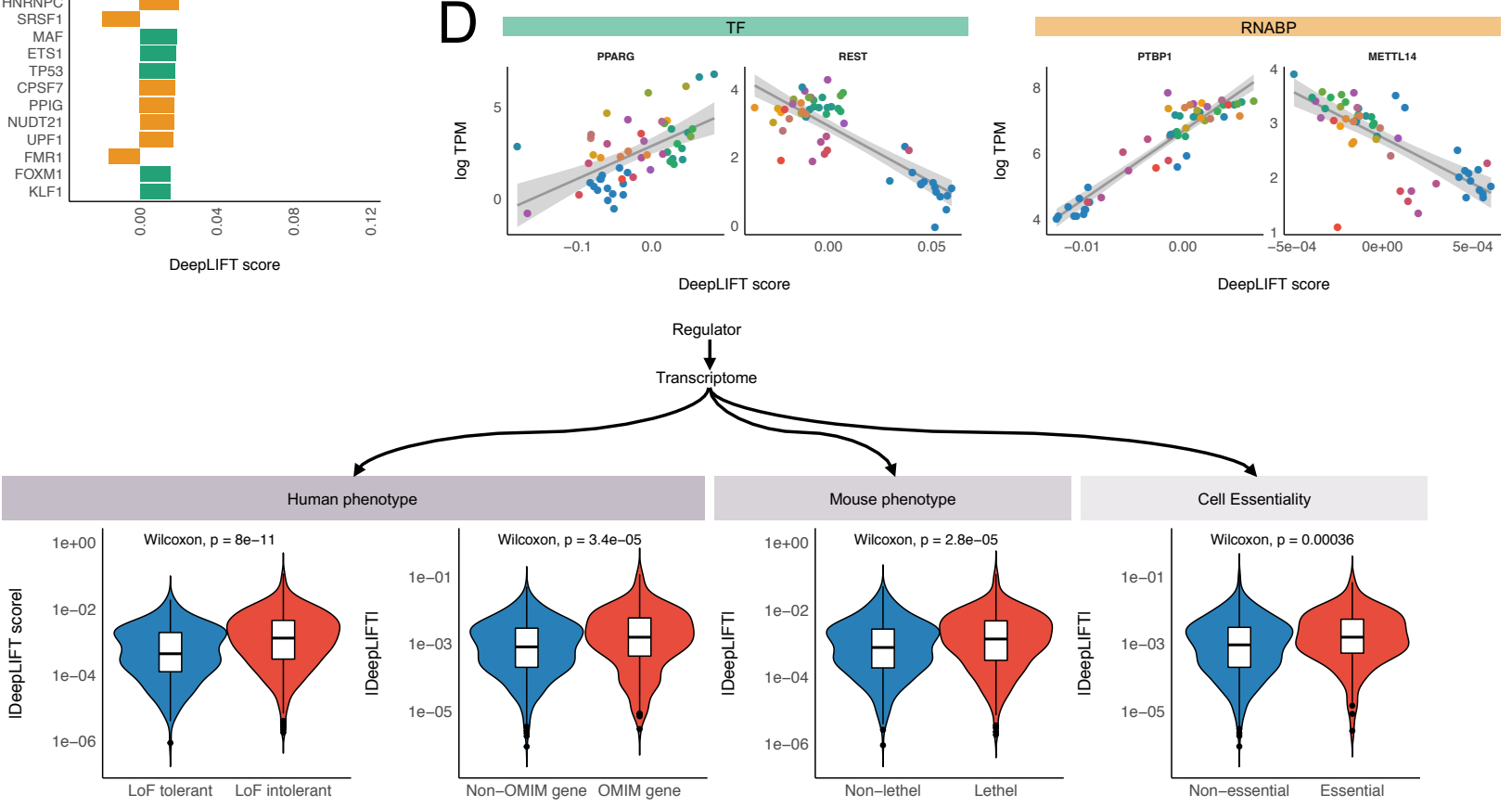

Regulator
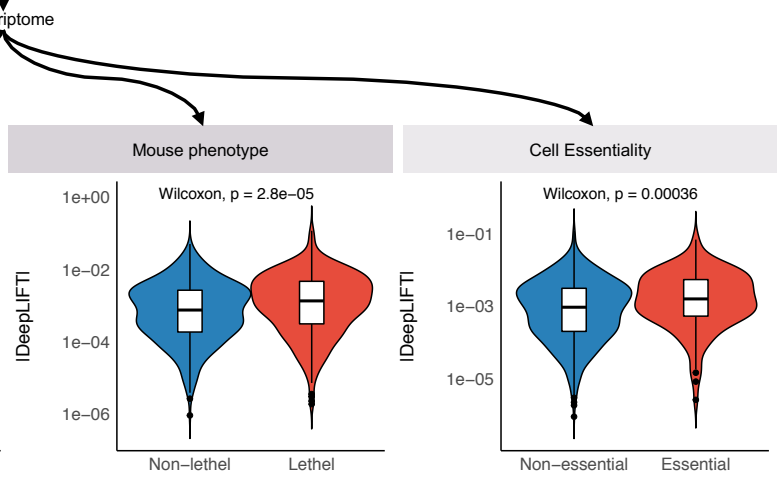

554 Figure 3. Identification and characterization of key predictors in the tissue-level models. (A) The enrichment of a regulator class in the key predictors for the median absolute expression levels. We ranked the regulators by the DeepLIFT scores and evaluated the enrichment of each regulator class. We used the pre-ranked gene set enrichment analysis (GSEA) algorithm with 10,000 permutations to compute enrichment scores and statistical significance. (B) Top 30 key predictors 
558 for the median absolute expression levels. (C) Key predictive regulators for the tissue-specific transcriptomes. We 559 selected the top 5 key predictors for each tissue and their DeepLIFT scores were displayed as a heatmap. The ward 560 linkage method with the Euclidean distance was used to cluster tissues and predictors. (D) Example relationships between 561 the predictive importance for a regulator and its expression levels across tissues. (E) The overlap between the key 562 regulators for the median absolute expression levels and external functional gene sets. 


\section{Figure 4}

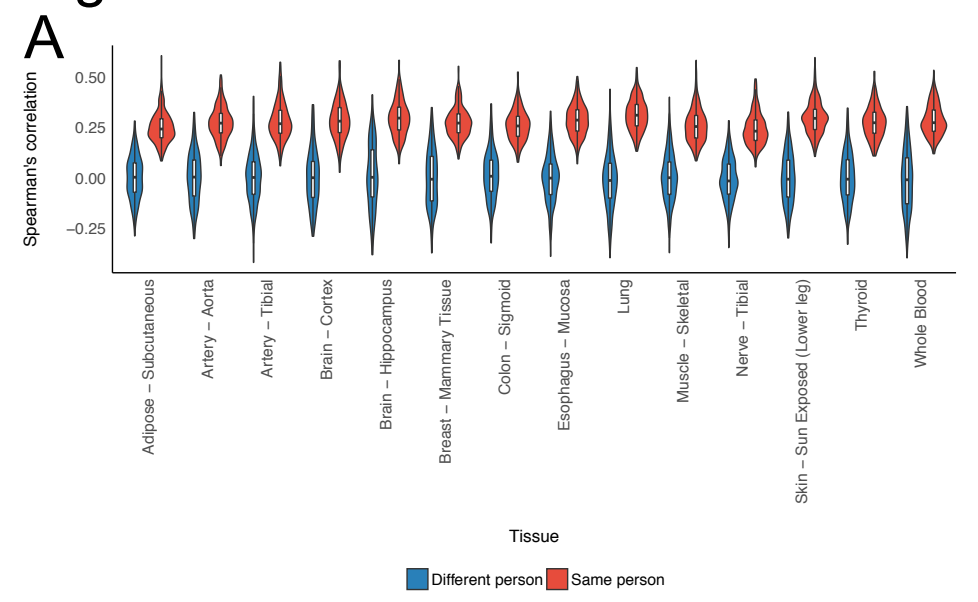

B
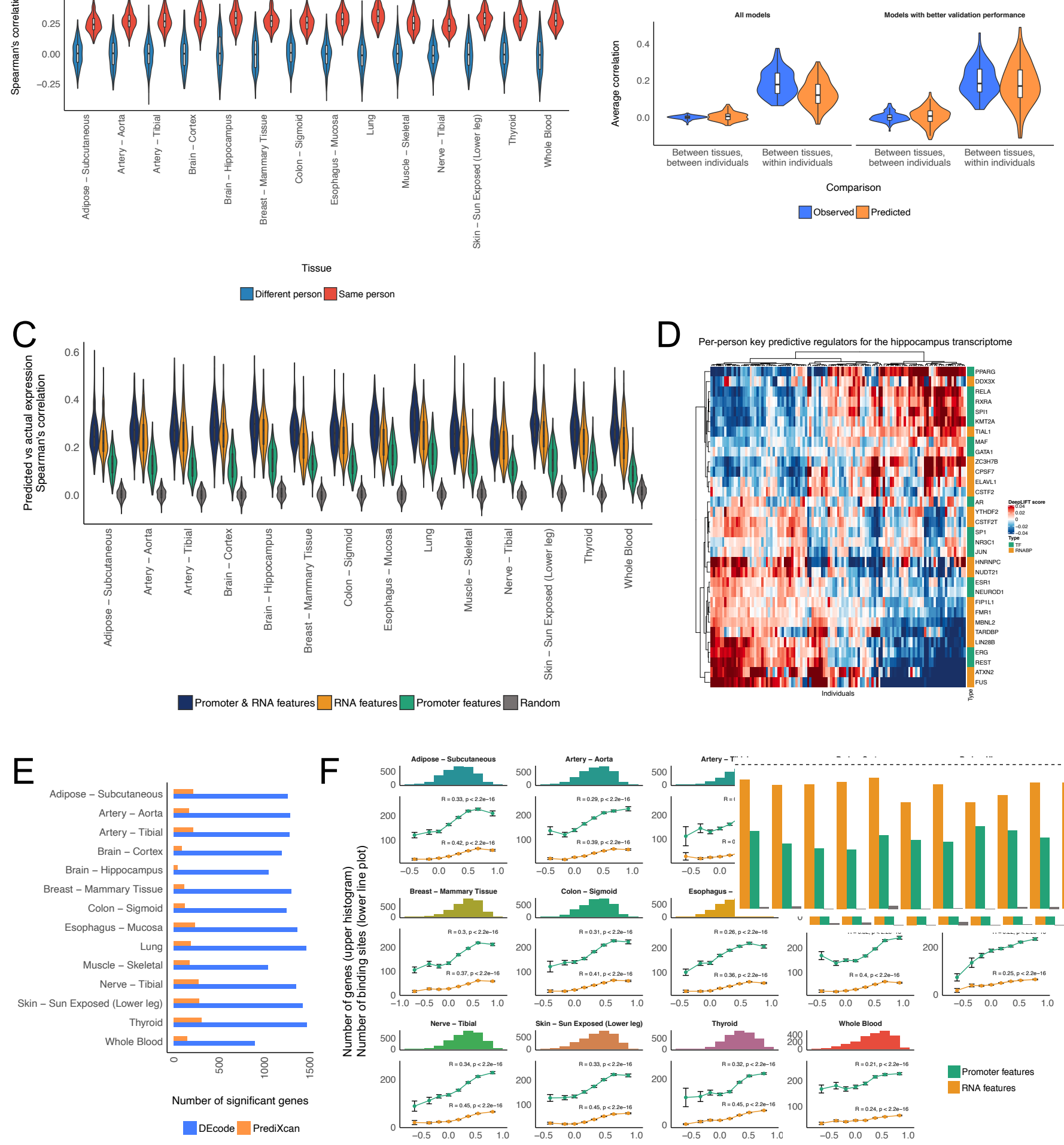

Per-gene prediction accuracy (Spearman's correlation)
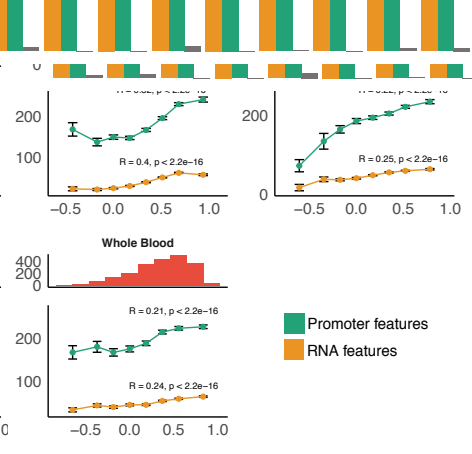

Figure 4. Performance of the person-specific models. (A) The predictive performances of the person-specific models for

the actual data from the same individuals and unrelated random individuals. (B) The person-specific models predicted person-specific expression shared across tissues. (C) The performances of the models trained with a distinct feature set. 
567 (D) Per-person key predictive regulators for the hippocampus transcriptome. We selected the top 5 key predictors of the 568 hippocampus transcriptome for each individual and their DeepLIFT scores were displayed as a heatmap. The ward 569 linkage method with the Euclidean distance was used to cluster tissues and predictors. (E) Comparison of per-gene 570 predictive accuracy between DEcode and PrediXcan. The number of genes that showed a positive Pearson's correlation 571 between predicted and actual gene expression levels at FDR 5\% was calculated for each method. Only the testing genes 572 on chromosome 1 were used for this comparison. (F) Per-gene prediction accuracy is associated with the number of 573 features present in RNAs and promoters. The histogram represents Pearson's correlations between the predicted and the 574 actual expression for each gene. The line plot shows the average number of RNA and promoter features of genes in each 575 bin of the histogram. Spearman's correlation between the number of features and per-gene correlations is displayed in 576 the line plot. The error bars indicate standard errors. 
Figure 5

A
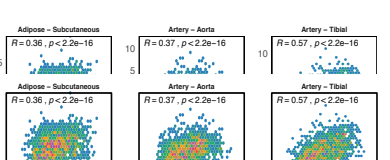

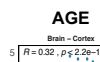
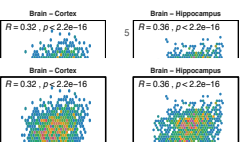

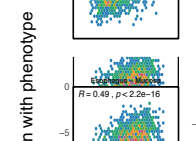
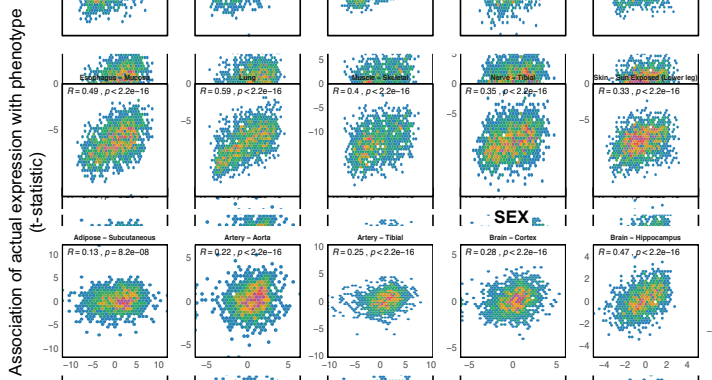

SEX
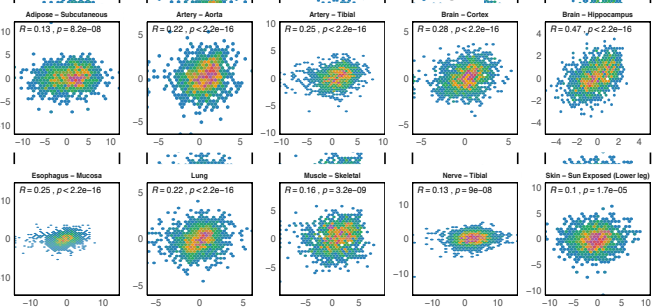

Association of predicted expression with phenotype (t-statistic)

AGE
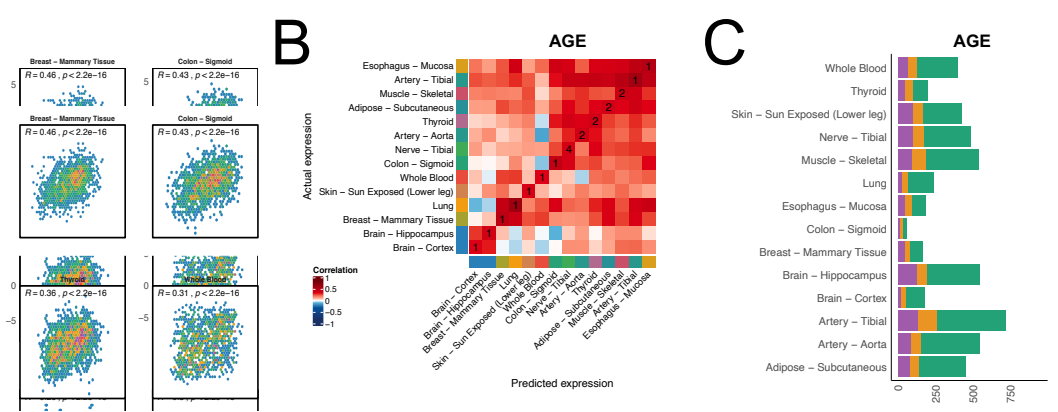

SEX
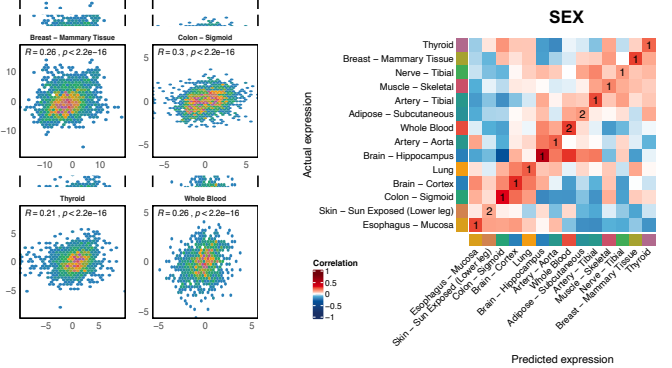

Figure 5. Application of the person-specific models to analyze phenotype-related gene signatures. (A) The scatter plots showing the relations between the associations of genes with age and sex using predicted expression and those using the actual expression. Spearman's correlation between t-statistics using the predicted and the actual gene expression is displayed in the scatter plot. (B) The pairwise Spearman's correlations between the predicted and the actual associations of genes with age and sex in all tissues. The numbers in diagonal elements of the heatmap indicate the ranks of similarity

583 of the predictions with the actual observations in the corresponding tissues. (C) The regulators whose DeepLIFT scores

584 were associated with age and sex. The Benjamini-Hochberg procedure was used to control the false discovery rate at $5 \%$

585 for each phenotype. 


\section{Figure 6}
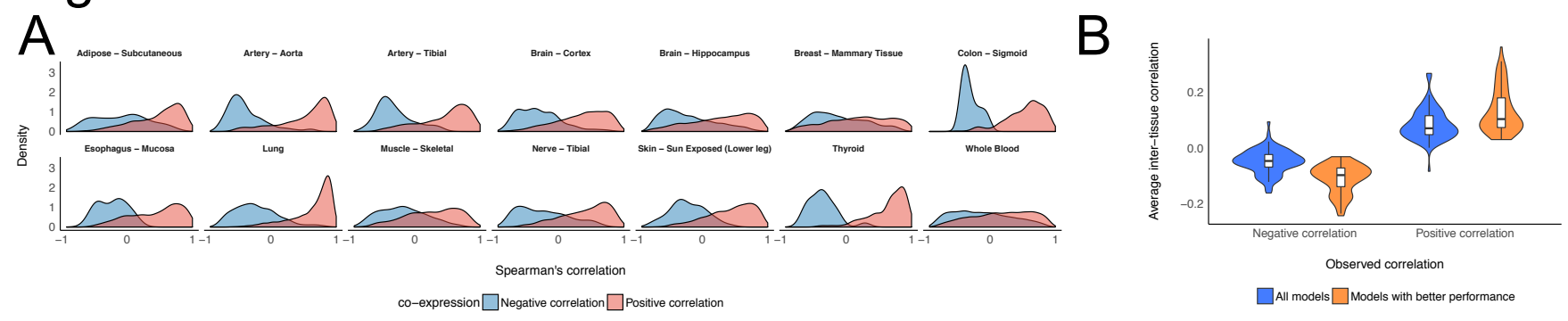

\section{C}

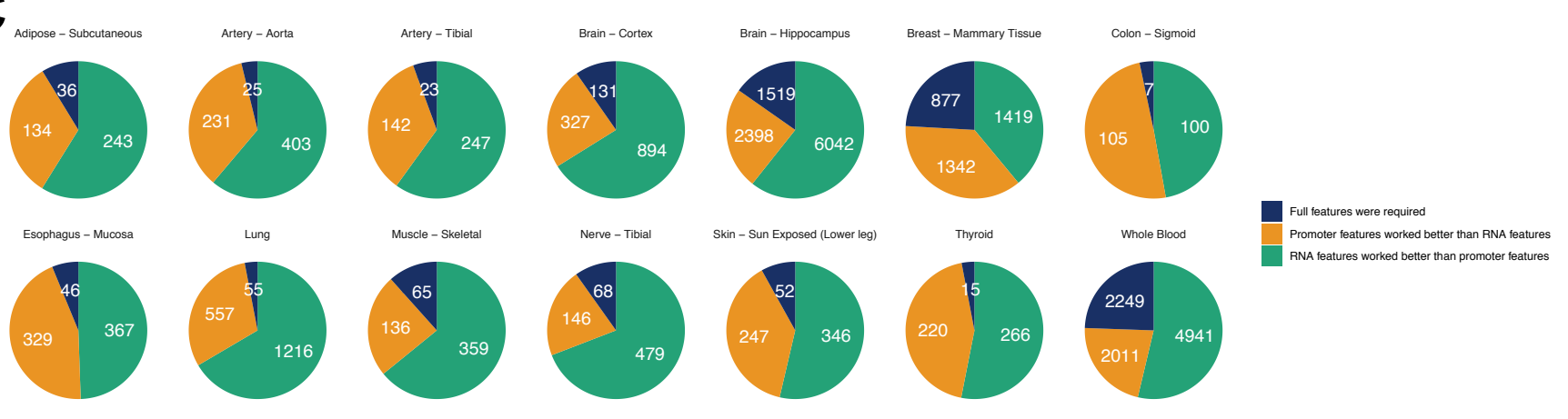

D

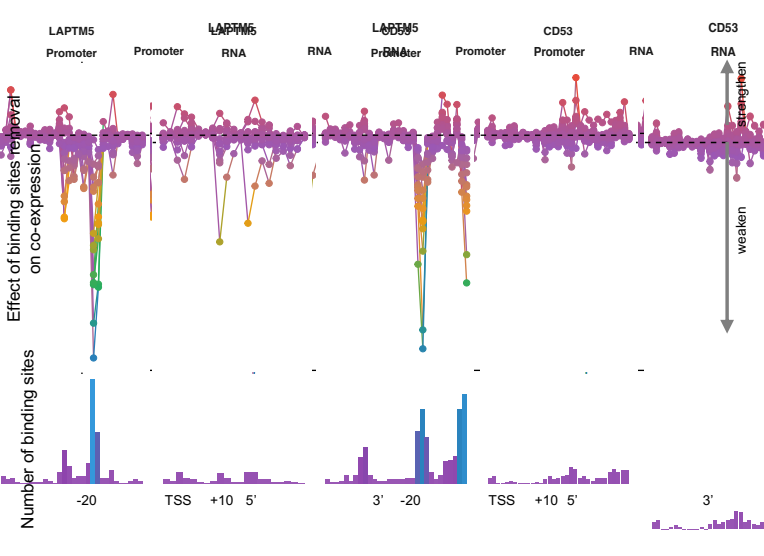

$\mathrm{F}$
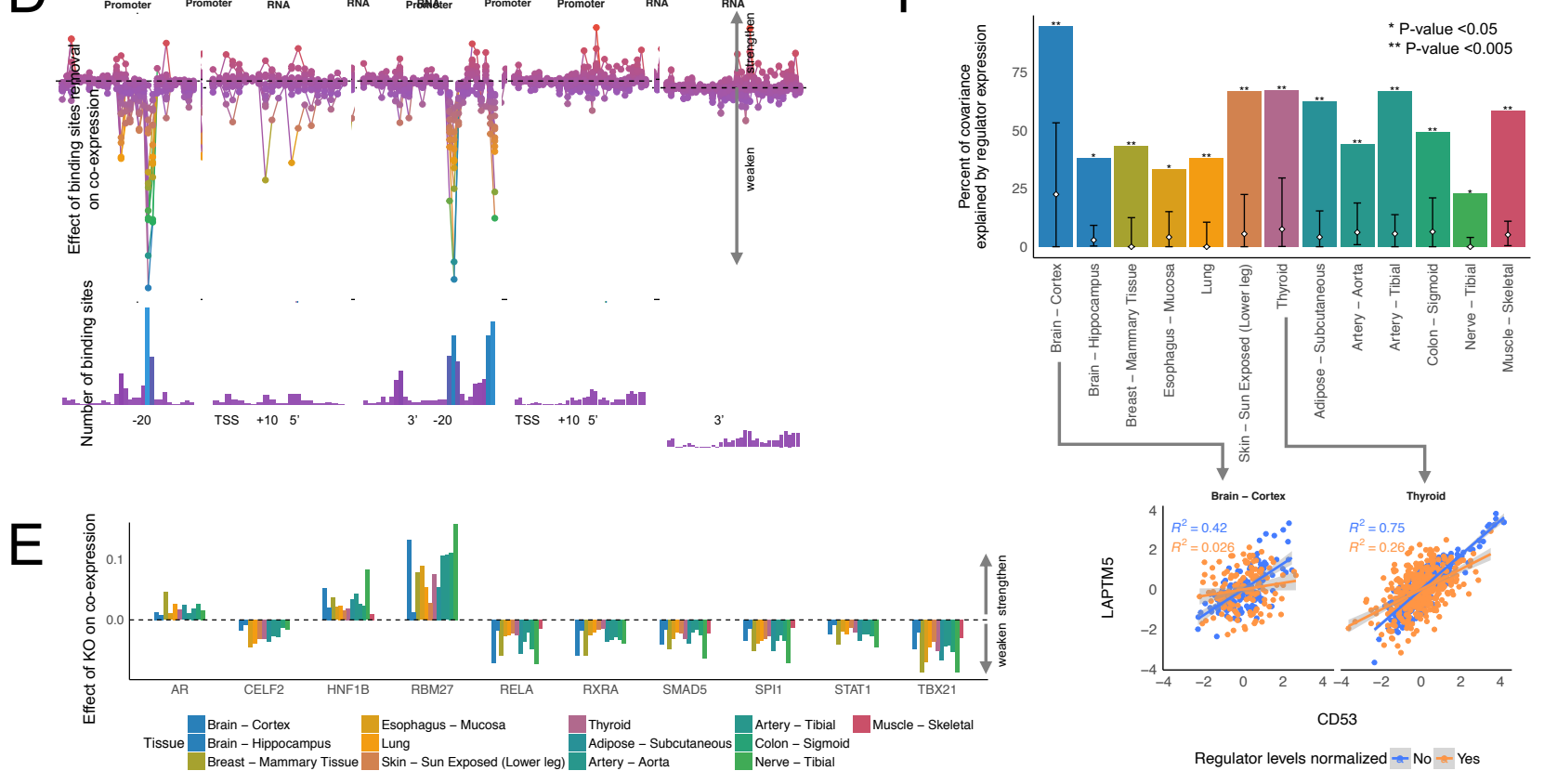

Regulator levels normalized $\approx$ No - Yes

Figure 6. Application of the person-specific models to investigate gene co-expression. (A) Co-expression relationships in the predicted gene expression. We defined the ground truth co-expression relationships as gene pairs with the absolute Spearman's correlation greater than 0.7 in the actual expression of the testing data. The density of Spearman's correlation between the co-expressed gene pairs in the predicted gene expression data was estimated using the density function in $\mathrm{R}$ with the Gaussian kernel. (B) Inter-tissue gene co-expression relationships in the predicted gene expression. We defined the ground truth co-expression relationships as gene pairs with the absolute Spearman's correlation greater than 0.5 in 
593 the actual expression of the testing data. We computed the average of Spearman's correlation of the inter-tissue co594 expressed gene pairs in each pair of tissues using the predicted expression from all models and the models whose 595 performances on validation data were greater than $50 \%$ percentile in each tissue. (C) The major feature types contributed 596 to the gene co-expression. We defined the gene pairs with the absolute Spearman's correlation greater than 0.3 and the 597 sign of the correlation matched with one with the ground truth as the successfully predicted gene pairs. The successfully 598 predicted gene pairs of the model trained with the full set of features were split into three groups based on the performance 599 of the models trained with only RNA features or promoter features. (D) The effect of the binding site removal on co600 expression between LAPTM5 and CD53. We simulated gene expression profiles with random removals of the binding 601 sites in each gene 10,000 times and computed a correlation between LAPTM5 and CD53 for each simulation. Multiple 602 regression was used to estimate the effect of the binding site removal on the co-expression in each tissue. (E) The key 603 regulators for the co-expression between LAPTM5 and CD53. We simulated gene expression profiles with random KOs 604 of regulators in each gene 10,000 times and computed a correlation between LAPTM5 and CD53 for each simulation. 605 We used multiple regression to estimate the effect of the $\mathrm{KO}$ on the co-expression in each tissue and identified the 606 consensus regulators across tissues. (F) Percent of the co-expression relationship explained by the expression levels of 607 the key regulators. The white diamond and the error bars in the bar indicated the average and $95 \%$ percentile of the 608 percent of variance explained by randomly picked regulators, respectively. The scatter plots show the effect of the key 609 regulators on the co-expression. 
Figure 7

\section{A}

DE Prior Rank

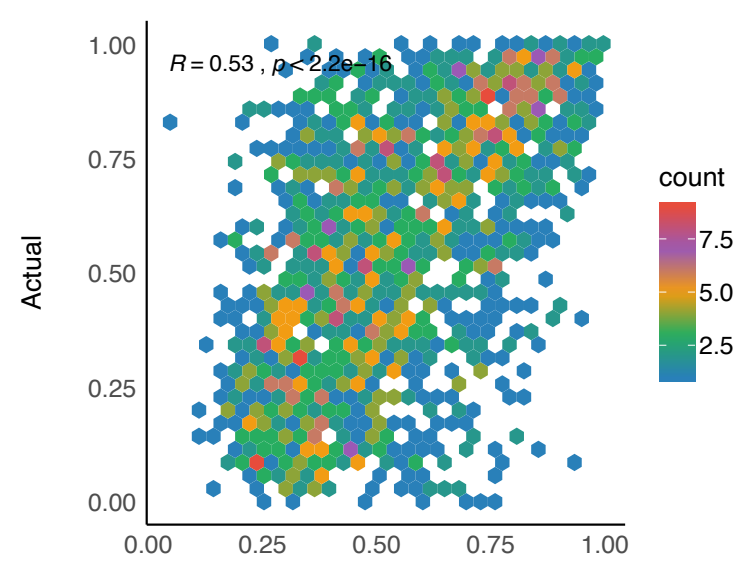

Predicted

\section{B}

Prediction of genes with DE Prior Rank $>0.9$

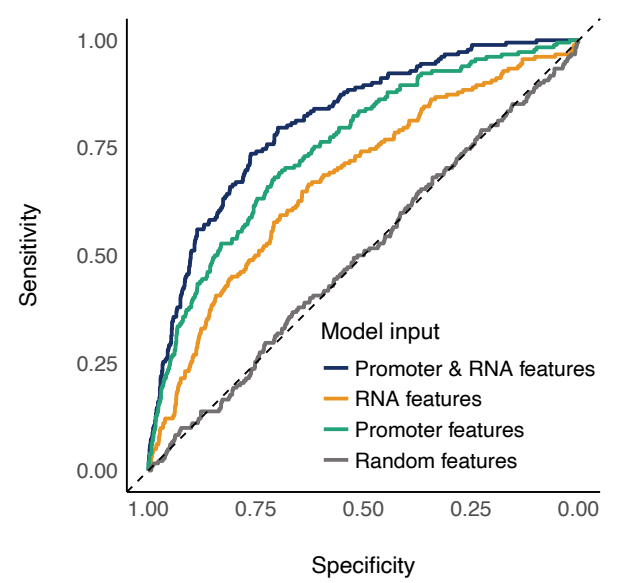

611 Figure 7. DEcode predicts gene's prior probability of differential expression. (A) The scatter plots showing the relations

612 between predicted and actual DE prior rank. The predicted logit of DE prior rank was converted to probability and

613 compared with actual DE prior rank with Spearman's correlation. (B) The performances of the models trained with a

614 distinct feature set. ROC represents the performance of model predicting genes with DE prior rank greater than 0.9 . 